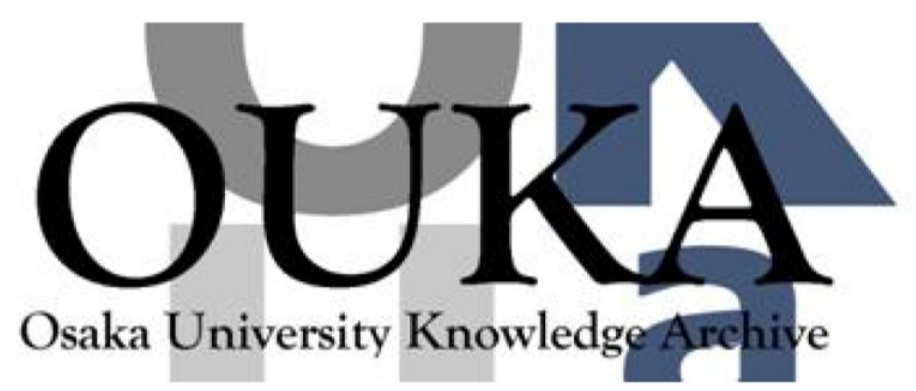

\begin{tabular}{|c|l|}
\hline Title & $\begin{array}{l}\text { Circular Dichroism of Optically Active } \\
\text { Poly(dialkylsilane) Aggregates in Microcapsules }\end{array}$ \\
\hline Author(s) & $\begin{array}{l}\text { Terao, Ken; Kikuchi, Noriaki; Sato, Takahiro et } \\
\text { al. }\end{array}$ \\
\hline Citation & Langmuir. 22(19) p. 7975-p. 7980 \\
\hline Issue Date & 2006-08-10 \\
\hline oaire:version & AM \\
\hline URL & $\begin{array}{l}\text { https://hdl.handle. net/11094/81828 } \\
\text { This document is the Accepted Manuscript } \\
\text { version of a Published Work that appeared in } \\
\text { final form in Langmuir, } 0 \text { American Chemical } \\
\text { Society after peer review and technical editing } \\
\text { by the publisher. To access the final edited } \\
\text { and published work see } \\
\text { https://doi.org/10.1021/la060810t. }\end{array}$ \\
\hline rights \\
\hline Note & \begin{tabular}{l} 
\\
\hline
\end{tabular} \\
\hline
\end{tabular}

Osaka University Knowledge Archive : OUKA

https://ir. Library. osaka-u. ac. jp/

Osaka University 


\title{
Circular Dichroism of Optically Active Poly(dialkylsilane) Aggregates in Microcapsules
}

\author{
Ken Terao, ${ }^{*}, \dot{\dagger}$ Noriaki Kikuchi,${ }_{\dagger}^{\dagger}$ Takahiro Sato, ${ }^{\ddagger}$ Akio Teramoto, $\|$ Michiya Fujiki, ${ }^{\S}$ and Toshiaki \\ Dobashi ${ }^{\dagger}$
}

Department of Biological and Chemical Engineering, Faculty of Engineering, Gunma University, 1-5-1 Tenjin-cho, Kiryu 376-8515, Japan; Department of Macromolecular Science, Graduate School of

Science, Osaka University, 1-1 Machikaneyama-cho, Toyonaka 560-0043, Japan; Department of Applied Chemistry, Faculty of Engineering, Ritsumeikan University, 1-1-1 Nojihigashi, Kusatsu 5258577, Japan; Graduate School of Materials Science, Nara Institute of Science and Technology, 8916-5 Takayama, Ikoma 630-0101, Japan.

* To whom correspondence should be addressed. E-mail: kterao@chem.sci.osaka-u.ac.jp (K. Terao)

$\dagger$ Gunma University.

$\$$ Osaka University.

$\|$ Ritsumeikan University.

$\S$ Nara Institute of Science and Technology.

Abstract: Poly( $n$-hexyl-(S)-3-methylpentylsilane) aggregates confined in microcapsules to keep the aggregation number and ranging in average polymer mass $m_{\mathrm{p}}$ in a microcapsule from $2 \times 10^{-16}$ to $2 \times 10^{-14}$ $\mathrm{g}$ were studied by circular dichroism measurements in ethanol (a non solvent) and tetrahydrofuran (an associative solvent at low temperature) at various temperatures. The weight-average molecular weight $M_{\mathrm{w}}$ and the polydispersity index PDI (the ratio of $M_{\mathrm{w}}$ to the number-average molecular weight) of the polysilane sample were $6.6 \times 10^{4}$ and 1.07 , respectively, and the average number of polymer molecule in each capsule was estimated to be $1.9 \times 10^{3}$ for $m_{\mathrm{p}}=2 \times 10^{-16} \mathrm{~g}$ and $1.7 \times 10^{5}$ for $m_{\mathrm{p}}=2 \times 10^{-14} \mathrm{~g}$. The size of each aggregate did not affect the optical activity because the circular dichroism thus obtained was proportional to $m_{\mathrm{p}}$ at the same condition in the investigated $m_{\mathrm{p}}$ range; on the other hand, the peak height of the circular dichroism in tetrahydrofuran had a significant hysteresis between 0 and $25^{\circ} \mathrm{C}$. Moreover, the circular dichroism appreciably reflected the prepared method, i.e. temperature and solvent; in other words, the aggregates memorized the initial condition in their stacking structures.

\section{Introduction}

The optical rotation and the circular dichroism (CD) are applied to determine the helical structure of polymer backbone for $\sigma$ or $\pi$ conjugate polymers, e.g. polyisocyanates ${ }^{1,2}$ and polysilanes ${ }^{3,4}$ because chiroptical effects are one of the local probes and usually insensitive to long range interactions. When two or more helical polymer chains overlap each other, that is, aggregates or thin films, it is expected that the circular dichroism is observed in terms of allocating polymer molecules anisotropically. Recently, Nakashima et al. and Koe et al. ${ }^{5,6}$ reported that aggregates of some poly[alkyl(alkylphenyl)]silanes bearing chiral side groups have much larger CD signal than those for molecularly dispersed system around the optical absorption band based on the Si-Si main chain, and the sign of the CD signal depends on the side chain, solvent, and/or temperature. Similar behavior was also found for chiral polythiophene aggregates. ${ }^{7,8,9}$

To clarify these phenomena, the size of the aggregates should be observed for various conditions, however we have recognized only a few works reported for both the optical activity and the average size of aggregates, ${ }^{10,11,12}$ as some aggregates frequently grow up too quickly to control their dispersibility during measurements of optical properties. One of the representative methods to control the size of aggregates is to apply self-assembled micelles consisting of block copolymers. Indeed, optical activity of the chiral aggregates was investigated using origo-thiophenes having origo-ethyleneglycol chains on the both ends in selective solvents. ${ }^{9,13,14,15}$ Furthermore, Sommerdijk et al. ${ }^{16}$ and Sanji et al. ${ }^{17,18}$ synthesized amphiphilic block copolymers including polysilanes and showed that the local conformation observed by UV-absorption for the size-controlled polysilane aggregates in the micelle is 
obviously different from the same polysilanes in molecularly dispersed systems; it is however laborious to apply the synthesis to polysilanes with chiral side chains. Recently, we developed a method to prepare size-controlled polysilane aggregates confined in polyethyleneglycol-grafted poly(ureaurethane) (PUU) microcapsules as shown in Figure 1 and we reported our preliminary results that the aggregates composed of poly[n-hexyl-(S)-3-methylpentylsilane] (PH3MPS) or poly[n-decyl- $(S)-2-$ methylbutylsilane] memorized the prepared temperature as CD signals. ${ }^{19}$ Similar behaviors were also found for thin films consisting of chiral polysilanes. ${ }^{20}$ Incidentally, an advantage investigating polymer aggregates with microcapsules is that the maximum number of aggregates can be easily controlled by the polymer concentration of the solution confined in the microcapsules. However, there were few experimental evidences about the effects of the aggregation number and the wall membrane of the microcapsules, suggesting that different-sized aggregates should be investigated in the same condition. In this study, we therefore prepared PUU microcapsules confining PH3MPS solution with a variety of polymer concentrations and measured CD spectra for PH3MPS aggregates prepared in a non-solvent at different temperatures and in an associative solvent in a wide temperature range to characterize the chiroptical properties of aggregates and aggregate-dissolution behavior.

\section{Experimental Section}

Materials. The well-fractionated PH3MPS sample ${ }^{21}$ F62 with the weight-average molecular weight being $6.60 \times 10^{4}$ was dissolved in isooctane to prepare the solutions with the polymer mass concentration $c_{\mathrm{p}}$ being $2.0 \times 10^{-4}, 6.0 \times 10^{-4}, 2.0 \times 10^{-3}$, and $6.0 \times 10^{-3} \mathrm{~g} \mathrm{~cm}^{-3}$. Takenate D120N $(70-80 \%$ trimethylolpropane carbamate with hexahydroxylylene diisocyanate in ethyl acetate) gifted from MitsuiTakeda Chemicals, Inc. was used as a wall-forming material. A polyethyleneglycol monomethyl ether (PEGME) sample with the molecular weight being 5000 was used as graft polymer chains on the microcapsules.

An adduct of Takenate D120N and PEGME was synthesized as follows; $40 \mathrm{~g}$ of Takenate D120 and $0.16 \mathrm{~cm}^{3}$ of tin(II)-2-ethylhexanoate as a catalyst were poured into PEGME solution of dried acetonitrile composed of $35 \mathrm{~g}$ of the polymer and $27 \mathrm{~g}$ of the solvent, and then the mixture was stirred for one hour at room temperature and for three hours at $50^{\circ} \mathrm{C}$. To obtain microemulsion confining the polysilane solution, $1.0 \mathrm{~g}$ of the resultant mixture, $1.0 \mathrm{~g}$ of Takenate $\mathrm{D} 120 \mathrm{~N}$, and $1 \mathrm{~cm}^{3}$ of the isooctane solution of PH3MPS F62 were added to $3.0 \mathrm{~g}$ of ethyl acetate, and then the resultant mixture was poured into $10 \mathrm{~g}$ of water and immersed vigorously for $10 \mathrm{~min}$ at 5000 or $15000 \mathrm{rpm}$ with an emulsifier (Excel Auto, Nihon Seiki Co.) in an ice bath. Then, the interfacial polymerization of the Takenate D120N proceeded at the interface of each emulsion with stirring the suspension in a beaker at $40^{\circ} \mathrm{C}$ for four hours without wrapping to remove ethyl acetate and acetonitrile. The volume fraction of the polysilane-isooctane solution in the resultant aqueous suspension was estimated to be 0.081 from the feed ratio. Two series of microcapsule suspension emulsified at 5000 and $15000 \mathrm{rpm}$ were designated as MC5k and MC15k, respectively.

To prepare polysilane aggregates in microcapsules, an appropriate amount $\left(0.010 \mathrm{~cm}^{3}\right.$ for MC15k and $0.025 \mathrm{~cm}^{3}$ for MC5k) of the microcapsule suspension which had been stored at room temperature $\left(20^{\circ} \mathrm{C}\right)$ was poured into $10 \mathrm{~cm}^{3}$ of ethanol at the prepared temperature $T_{\mathrm{p}}=20^{\circ} \mathrm{C}$ and $T_{\mathrm{p}}=-$ $78^{\circ} \mathrm{C}$ or into tetrahydrofuran (THF) at $T_{\mathrm{p}}=20^{\circ} \mathrm{C}$. It is known that the outer medium of PUU microcapsules replaces inner solvent when the inner and outer medium are miscible each other ${ }^{22,23,24,25,26,27,28}$ whereas polymer molecules stay in the capsule. ${ }^{19}$ The mass concentration $c_{\mathrm{ps}}$ of the polysilane sample per unit volume of the resulting suspension can be estimated to be $c_{\mathrm{ps}}=8.1 \times 10^{-5} c_{\mathrm{p}}$ for MC15k and $c_{\mathrm{ps}}=2.0_{3} \times 10^{-4} c_{\mathrm{p}}$ for MC5k, and the contents of water and isooctane in the resulting suspension were quite small, that is about $0.08 \%$ and $0.008 \%$ for $\mathrm{MC} 15 \mathrm{k}$ and $0.2 \%$ and $0.02 \%$ for MC5k, respectively.

Circular Dichroism (CD) Measurements. CD measurements for the microcapsules in ethanol at the measured temperature $T_{\mathrm{m}}$ between $10^{\circ} \mathrm{C}$ and $40^{\circ} \mathrm{C}$ and in THF at $T_{\mathrm{m}}$ between -10 and $40^{\circ} \mathrm{C}$ were made on a JASCO J720 spectropolarimeter combined with a Peltier temperature controller at a wavelength of the incident light between 250 and $400 \mathrm{~nm}$. The scanning condition was as follows: A bandwidth of $1 \mathrm{~nm}$, response of $1 \mathrm{~s}$, a scanning rate of $50 \mathrm{~nm} / \mathrm{min}$, and single or double accumulations. 
A quartz cell with the optical pass length being $1.0 \mathrm{~cm}$ was used and the suspension in the cell was stirred with a magnetic stirrer during measurements. Cooling and heating rate of a cell holder was less than $10^{\circ} \mathrm{C} / \mathrm{min}$ and each accumulation was begun about ten minutes after the temperature of the cell holder had stabilized. The optical shutter of the equipment was closed except during measurements to avoid decomposition of the Si-Si main chain of the polysilane sample.

\section{Results and Discussions}

Characteristics of Microcapsules Confining the Polysilane Sample. The number average outer diameter $d_{\mathrm{o}, \mathrm{n}}$ and the ratio $d_{\mathrm{i}} / d_{\mathrm{o}}$ of inner to outer diameter for the microcapsule MC5k series were reported to be $2.0 \mu \mathrm{m}$ and 0.9 , respectively, in our previous paper. ${ }^{22}$ In addition, the $d_{\mathrm{o}, \mathrm{n}}$ value for the microcapsule MC15k series in water was determined to be $1.4 \mu \mathrm{m}$ by a microscopic observation; we note that $d_{\mathrm{i}} / d_{\mathrm{o}}$ is almost independent of $d_{\mathrm{o}}$ when the volume ratio of the core medium to the wallforming material has a constant value. ${ }^{22,29}$ The number-average inner diameter $d_{\mathrm{i}, \mathrm{n}}$ was estimated to be $1.26 \mu \mathrm{m}$ for MC15k and $1.8 \mu \mathrm{m}$ for MC5k. The mass $m_{\mathrm{p}}$ and the average molecular number $n_{\mathrm{p}}$ for the polysilane sample in a microcapsule were therefore estimated to be $\pi d_{\mathrm{i}, \mathrm{n}}{ }^{3} c_{\mathrm{p}} / 6$ and $m_{\mathrm{p}} N_{\mathrm{A}} / M_{\mathrm{w}}$, respectively, where $c_{\mathrm{p}}, N_{\mathrm{A}}$ and $M_{\mathrm{w}}$ are polysilane mass concentration of the confined isooctane solution, the Avogadro constant, and weight-average molecular weight for the polysilane sample, that is $6.6 \times 10^{4} .{ }^{21}$ The obtained $m_{\mathrm{p}}$ and $n_{\mathrm{p}}$ values for each sample are summarized in Table 1.

Wavelength dependences of the differential absorption $\Delta A$ between microcapsule suspensions with and without polysilane in $\mathrm{THF}$ at $40^{\circ} \mathrm{C}$ are illustrated in Figure 2; we note that the polysilane sample is molecularly dispersed in THF at $40^{\circ} \mathrm{C}$. UV spectra at other conditions we measured are fairly the same as the data shown in this figure, indicating that the dihedral angle of Si-Si main chain in aggregates molecule is not significantly different from that in solution. Whereas the extinction coefficient $\varepsilon_{\max }$ at the peak was determined to be $1.80 \times 10^{4}$ (Si-unit) $)^{-1} \mathrm{dm}^{3} \mathrm{~cm}^{-1}$ for PH3MPS F62 in THF at $40^{\circ} \mathrm{C}$, the apparent extinction coefficient $\varepsilon_{\text {max,app }}$ of $\varepsilon_{\text {max }}$ are determined from the peak height in Figure 2 to be $1.3{ }_{1} \times 10^{4}$ (Si-unit) ${ }^{-1} \mathrm{dm}^{3} \mathrm{~cm}^{-1}$ and $3.4_{8} \times 10^{3}$ (Si-unit) $)^{-1} \mathrm{dm}^{3} \mathrm{~cm}^{-1}$ for MC15k and MC5k, respectively. Thus, the ratio of $\varepsilon_{\max , a p p}$ to $\varepsilon_{\max }$ are estimated to be $0.72{ }_{8}$ for MC15k and $0.19_{3}$ for MC5k, suggesting that the MC15k sample is more suitable to obtain the absolute CD value; we note that the small $\varepsilon_{\text {max,app }} / \varepsilon_{\max }$ value for MC5k is due to the higher turbidity of microcapsule suspension.

Aggregates in Ethanol. The CD spectra in ethanol at different concentrations $c_{\mathrm{p}}$ show a bisigned Cotton effect and are similar in shape as illustrated in Figure 3; note that the CD in this figure has been corrected with the ratio $\varepsilon_{\text {max,app }} / \varepsilon_{\max }$. The intermediate wavelengths $\approx 320 \mathrm{~nm}$ of plus and minus peaks are fairly the same as those for the single peak in isooctane solution. ${ }^{30}$ It is known that the bisignate $\mathrm{CD}$ is an aspect for chiral PH3MPS aggregates. ${ }^{19}$ Therefore, it was considered that the bisignate $C D$ from aggregates is due to super-helical stacking of polysilane main chains, ${ }^{5,6}$ as the bisignate $\mathrm{CD}$ is a characteristic of exciton coupling between neighbor transition dipole moments in chiral configurations. ${ }^{31}$ While the peak height slightly decreased with rising temperature, the difference was only less than $10 \%$ between 10 and $40^{\circ} \mathrm{C}$.

To compare the strength of $\mathrm{CD}$ spectra on various conditions, the differential value $\triangle C D$ between the highest $\mathrm{CD}$ around $\lambda=325 \mathrm{~nm}$ and the lowest $\mathrm{CD}$ at about $\lambda=310 \mathrm{~nm}$ on each $\mathrm{CD}$ spectrum was introduced. The obtained $\triangle C D$ values plot against $c_{\mathrm{p}}$ and $m_{\mathrm{p}}$ in Figure 4 except for some samples with low $c_{\mathrm{p}}$ because of too small CD signals; we note that $\mathrm{CD}$ due to the helical structure of the main chain does not affect the total $\mathrm{CD}$ spectra because the peak height is calculated to be about only $0.08 \mathrm{mdeg}$ for MC5k-6 and $0.03 \mathrm{mdeg}$ for MC15k-6 in isooctane at $25^{\circ} \mathrm{C} .{ }^{30} \triangle C D$ is almost proportional to $m_{\mathrm{p}}$ in the $m_{\mathrm{p}}$ range illustrated in Figure 4; indicating that the $\triangle C D$ reflects only the average of local structures in the aggregates and the local structure is insensitive to the wall membrane of microcapsules.

Figure 4 also shows that the differential circular dichroism $\triangle C D_{-78}$ at $T_{\mathrm{p}}=-78^{\circ} \mathrm{C}$ (the prepared temperature, see the experimental section) are obviously larger than that $\Delta C D_{20}$ at $T_{\mathrm{p}}=20^{\circ} \mathrm{C}$ and the ratio of $\triangle C D_{-78}$ to $\triangle C D_{20}$ are estimated from the solid lines to be 3.9 and 3.1 for $\mathrm{MC} 15 \mathrm{k}$ and $\mathrm{MC} 5 \mathrm{k}$, 
respectively. The difference $\Delta \Delta \varepsilon$ of the molar circular dichroism, $\Delta \varepsilon$, defined by $\Delta \Delta \varepsilon=\Delta C D / 33 c_{\mathrm{ps}}$ are calculated to be 335 and 85 (Si-unit) $)^{-1} \mathrm{dm}^{3} \mathrm{~cm}^{-1}$ for MC15k at $T_{\mathrm{p}}=-78$ and $20^{\circ} \mathrm{C}$, respectively. These $\Delta \Delta \varepsilon$ values are much larger than $\Delta \varepsilon=17$ and 1.2 (Si-unit) $)^{-1} \mathrm{dm}^{3} \mathrm{~cm}^{-1}$ at the peak in a good solvent (isooctane) at -75 and $25^{\circ} \mathrm{C},{ }^{30}$ respectively; we note that the absolute $\triangle C D$ values for MC5k are about $30 \%$ smaller than those for MC15k because of difficulty to estimate $\varepsilon_{\text {app,max }}$ accurately due to the small value of $\varepsilon_{\max , a p p}$ for MC5k. The proximity of the ratio $\triangle C D_{-78} / \Delta C D_{20}$ for the two microcapsules indicates that the ratio is not significantly affected by the difference in the size of the microcapsules. Therefore, the $T_{\mathrm{p}}$ dependence of $\triangle C D$ and the proportionality relation between $\triangle C D$ and $m_{\mathrm{p}}$ for each microcapsule suggest that $T_{\mathrm{p}}$ is memorized in the local structure in aggregates as $\Delta \Delta \varepsilon$. In addition, it seems reasonable to suppose that the larger $\triangle C D$ at lower $T_{\mathrm{p}}$ is associated with the enantiomer excess of each polymer backbone: The main chain of PH3MPS F62 at $-78^{\circ} \mathrm{C}$ is composed of almost single-handed helix and the persistence length $q$ of the polymer is $15 \mathrm{~nm}$ even at $-27^{\circ} \mathrm{C}$, whereas the same polymer chain at $20^{\circ} \mathrm{C}$ has about 13 helix reversals per molecule and lower $q$, that is, $6 \sim 7 \mathrm{~nm} .^{21,30}$ This suggestion is also supported by the following experimental results: $T_{\mathrm{p}}$ dependence of the poly[ $n$-decyl$(S)$-2-methylbutylsilane] aggregates having highly rigid main chain ${ }^{32,33}$ and almost single-handed screw $^{34}$ was appreciably weaker than that for PH3MPS. ${ }^{19}$

Aggregates in Tetrahydrofuran (THF). As CD spectra for MC15k-6 in THF illustrated in Figure 5a resemble those in ethanol, the same definition of $\triangle C D$ was introduced to discuss CD intensity in THF. The $\triangle C D$ value for $\mathrm{MC} 15 \mathrm{k}$ in $\mathrm{THF}$ at $20^{\circ} \mathrm{C}$ has a small value, that is, $11.3 \mathrm{mdeg}$. The value of $\triangle C D$ increases gradually between 20 and $10^{\circ} \mathrm{C}$ with falling temperature, rapidly between 10 and $0^{\circ} \mathrm{C}$, gently below $0^{\circ} \mathrm{C}$, and reaches to $114 \mathrm{mdeg}$ at $-10^{\circ} \mathrm{C}$ as shown by open circles in Figure $5 \mathrm{~b}$. After annealed at $40^{\circ} \mathrm{C}, \triangle C D$ illustrated in Figure $6 \mathrm{~b}$ vanishes at the temperature higher than $10^{\circ} \mathrm{C}$ in the cooling process from $40^{\circ} \mathrm{C}$ due to dissociation of the polymer aggregate, increases extremely below $10^{\circ} \mathrm{C}$, and reaches to about $240 \mathrm{mdeg}$ at $0^{\circ} \mathrm{C}$. The open circles for $\triangle C D$ measured every $5 \mathrm{~K}$ from $20^{\circ} \mathrm{C}$ or $40^{\circ} \mathrm{C}$ almost corresponds to filled circles which was cooled directly from $20^{\circ} \mathrm{C}$ or $40^{\circ} \mathrm{C}$ to $0^{\circ} \mathrm{C}$ as shown in panels (a) and (b) in Figures 5 or 6, respectively; indicating that the cooling rate chosen for this study does not affect $\triangle C D$ of the polysilane aggregates. The difference of $\triangle C D$ at $0^{\circ} \mathrm{C}$ for Figures 5 and 6 will be described later.

Figure 7 shows $c_{\mathrm{p}}$ and $m_{\mathrm{p}}$ dependence of $\triangle C D$ for all samples in THF at typical temperatures on heating and cooling processes: Open and filled circles denote the data at $0^{\circ} \mathrm{C}$ on the first and second cooling, and filled triangles, open squares, and open triangles denote those at $20^{\circ} \mathrm{C}$ on first cooling (the initial condition), first heating, and second heating; we note that the temperature was changed as the following order: $20,-10,40,0$, and $40^{\circ} \mathrm{C}$. Straight lines illustrated in the figure represent the data points for each condition and have a slope of unity, indicating that $\Delta C D / m_{\mathrm{p}}$ for each condition is independent of $m_{\mathrm{p}}$. Indeed, the temperature variations of $\Delta \Delta \varepsilon$ for MC15k samples with different $m_{\mathrm{p}}$ in THF are fairly fitted by a universal curve as illustrated in Figure 8. Furthermore, from the solid lines in

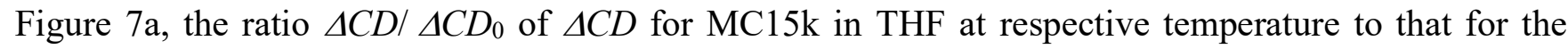
initial condition (at $20^{\circ} \mathrm{C}$ ) is determined to be 7.9 at $0^{\circ} \mathrm{C}$ on first cooling, 3.8 at $20^{\circ} \mathrm{C}$ on first heating, 20 at $0^{\circ} \mathrm{C}$ on second cooling, and 5.7 at $20^{\circ} \mathrm{C}$ on second heating. Solid lines in Figure $7 \mathrm{~b}$ drawn to have the same $\triangle C D / \triangle C D_{0}$ as those for $\mathrm{MC} 15 \mathrm{k}$ represent the data points almost quantitatively. These results confirm the above-mentioned suggestion; that is to say, the local structure in each aggregate is not influenced by the size of aggregates in the current $m_{\mathrm{p}}$ range.

Although fully temperature-reversible $\mathrm{CD}$ spectra was observed for molecularly dispersed PH3MPS in solution, ${ }^{30} \Delta \Delta \varepsilon$ in Figure 8 decreases gradually from $10^{\circ} \mathrm{C}$ and vanishes at about $30^{\circ} \mathrm{C}$ in heating process and then $\Delta \Delta \varepsilon$ vanishes even at $15^{\circ} \mathrm{C}$ in the second cooling process. This thermal hysteresis suggests that the aggregate-dissociation process is irreversible due to the formation of interchain structure. Similar hysteresis phenomena are also seen in some physical sol-gel transitions, ${ }^{35}, 36$ an aggregate-dissociation transition, ${ }^{37}$ and a coil-globule transition of single molecule. ${ }^{38,39}$ 
Each microcapsule confining F62 in THF has an unmistakable CD due to the aggregates even at the initial condition $\left(20^{\circ} \mathrm{C}\right)$ as illustrated in Figures 5 and 8, indicating that the polymer chains is weakly aggregated. However, only a very small CD signal reflecting the helical structure of the main chain was

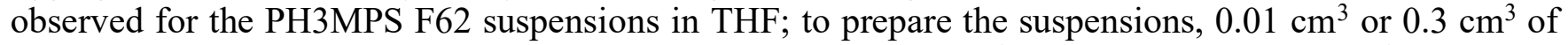
F62-isooctane solutions with the concentration being $0.001 \mathrm{~g} \mathrm{~cm}^{-3}$ had been added to $5 \mathrm{~cm}^{3}$ of THF at $20^{\circ} \mathrm{C}$. Taking into account the no $\mathrm{CD}$ signal at $20^{\circ} \mathrm{C}$ for all investigated microcapsules annealed at $40^{\circ} \mathrm{C}$, it seems that the wall membrane does not affect significantly the aggregation behavior; therefore, the following reason can be pointed out to explain the presence of $\triangle C D$ at the initial condition: The composition of water (a non solvent of PH3MPS) in microcapsule possibly became high enough to form aggregates of the polysilane during replacing solvents because the microcapsules were surrounded by water before addition to THF, and then the aggregates did not completely dissolve even in almost pure THF at $20^{\circ} \mathrm{C}$. A further important point is that $\triangle C D$ at $0^{\circ} \mathrm{C}$ without annealing is about 0.4 times as large as that at the same temperature after annealing as shown in Figure 7 . In addition, $\triangle C D$ at $0^{\circ} \mathrm{C}$ after annealing is much larger than that prepared in ethanol, indicating that the regularity of chiral structure in temperature-induced aggregates is higher than that induced by solvent replacement; indeed polymer-polymer interaction factors accompanying temperature variation changes usually much milder than that due to solvent exchange.

\section{Conclusions}

In the present study, we prepared different-sized microcapsules confining different mass of a $\operatorname{poly}(n$-hexyl-(S)-3-methylpentylsilane) sample to control the aggregation number and we measured size dependence of circular dichroism for the aggregates in ethanol (a non-solvent) and in tetrahydrofuran (an associative solvent) at various temperatures. Temperature-induced aggregation-dissolution behavior shows a typical hysteresis whereas the local structure in aggregates is irrespective of polymer mass per microcapsule. Furthermore, the circular dichroism significantly depends on the prepared condition, i.e. temperature and solvent, unless the aggregates were molecularly dissolved at high temperature; in other words, the prepared condition was memorized as the local structure in aggregates and can be observed by the circular dichroism.

Acknowledgement. The authors are grateful to Dr. Kimio Ichikawa at Fuji Photo Film Co. for valuable discussions. This research was partially supported by Grant-in-Aid for Young Scientists (B) from Ministry of Education, Culture, Sports, Science, and Technology (MEXT) under grant number of 16750109 .

\section{References}

(1) Green, M. M. In Circular Dichroism-Principles and Applications, 2nd ed; N. Berova, K. Nakanishi, R. W. Woody Eds., Wiley-VCH: New York, 2000.

(2) Teramoto, A. Prog. Polym. Sci. 2001, 26, 667-720.

(3) Fujiki, M.; Koe, J. R.; Terao, K.; Sato, T.; Teramoto, A.; Watanabe, J. Polym. J. 2003, 35, $297-$

344.

(4) Sato, T.; Terao, K.; Teramoto, A.; Fujiki, M. Polymer 2003, 44, 5477-5495.

(5) Nakashima, H.; Koe, J. R.; Torimitsu, K.; Fujiki, M. J. Am. Chem. Soc. 2001, 123, 4847-4848.

(6) Peng, W.; Motonaga, M.; Koe, J. R. J. Am. Chem. Soc. 2004, 126, 13822-13826.

(7) Langeveld-Voss, B. M. W.; Christiaans, M. P. T.; Janssen, R. A. J.; Meijer, E. W.

Macromolecules 1998, 31, 6702-6704.

(8) Langeveld-Voss, B. M. W.; Waterval, R. J. M.; Janssen, R. A. J.; Meijer, E. W. Macromolecules 1999, 32, 227-230.

(9) Leclere, P.; Surin, M.; Viville, P.; Lazzaroni, R.; Kilbinger, A. F. M.; Henze, O.; Feast, W. J.; Cavallini, M.; Biscarini, M.; Schenning, A. P. H. J.; Meijer, E. W. Chem. Mater. 2004, 16, 4452-4466.

(10) Reidy, M. P.; Green, M. M. Macromolecules 1990, 23, 4225-4234.

(11) Yue, S.; Berry, G. C.; Green, M. M. Macromolecules 1996, 29, 6175-6182. 
(12) Guenet, J.-M.; Jeon, H. S.; Khatri, C.; Jha, S. K.; Balsara, N. P.; Green, M. M.; Brulet, A.; Thierry, A. Macromolecules 1997, 30, 4590-4596.

(13) Brustolin, F.; Goldoni, F.; Meijer, E. W.; Sommerdijk, N. A. J. M. Macromolecules 2002, 35 , 1054-1059.

(14) Schenning, A. P. H. J.; Kilbinger, A. F. M.; Biscarini, M.; Cavallini, M.; Cooper, H. J.; Derrick, P. J.; Feast, W. J.; Lazzaroni, R.; Leclere, Ph.; Mcdonell, L. A. J.; Meijer, E. W.; Meskers, S. C. J. Am. Chem. Soc. 2002, 124, 1269-1275.

(15) Henze, O.; Feast, W. J.; Gardebien, F.; Jonkheijm, P.; Lazzaroni, R.; Leclere, Ph.; Meijer, E. W.; Schenning, A. P. H. J. J. Am. Chem. Soc. 2006, 128, 5923-5929.

(16) Sommerdijk, N. A. J. M.; Holder, S. J.; Hiorns, R. C.; Jones, R. G.; Nolte, R. J. M. Macromolecules 2000, 33, 8289-8294.

(17) Sanji, T.; Kitayama, F.; Sakurai, H. Macromolecules 1999, 32, 5718-5720.

(18) Sanji, T.; Nakatsuka, Y.; Kitayama, F.; Sakurai, H. Chem. Comm. 1999, 2201-2202.

(19) Terao, K.; Mori, Y.; Dobashi, T.; Sato, T.; Teramoto, A.; Fujiki, M. Langmuir 2004, 20, 306308.

(20) Ohira, A.; Kunitake, M.; Fujiki, M.; Naito, M.; Saxena, M. Chem. Mater. 2004, 16, 3919-3923.

(21) Terao, K.; Terao, Y.; Teramoto, A.; Nakamura, N.; Fujiki, M.; Sato, T. Macromolecules 2001, $34,4519-4525$.

(22) Terao, K.; Ohsawa, A.; Mori, Y.; Narita, T.; Ichikawa, K.; Dobashi, T. Colloids Surf., B 2004, 37, 129-132.

(23) Yamamoto, T.; Dobashi, T.; Kimura, M.; Chang, C. P. Colloids Surf., B 2002, 25, 305-311.

(24) Sato, T.; Yamamoto, T.; Shibako, S.; Ichikawa, K.; Dobashi, T. J. Membr. Sci. 2003, 213, $25-31$.

(25) Chang, C. P.; Yamamoto, T.; Kimura, M.; Sato, T.; Ichikawa, K.; Dobashi, T. J. Controlled Release 2003, 86, 207-211.

(26) Chang, C. P.; Kimura, M.; Yamamoto, T.; Nobe, M.; Dobashi, T. Colloids Surf., B 2003, 30, 123-127.

(27) Furukawa, T.; Hung, S.; Yamamoto, T.; Terao, K.; Ichikawa, K.; Dobashi, T. Trans. Mater. Res. Soc. Jpn. 2003, 28, 989-992.

(28) Chang, C. P.; Chang, J. C.; Ichikawa, K.; Dobashi, T. Colloids Surf., B 2005, 44, 183-186.

(29) Dobashi, T.; Furukawa, T.; Narita, T.; Shimofure, S.; Ichikawa, K.; Chu, B. Langmuir 2001, 17, 4525-4528.

(30) Terao, K.; Terao, Y.; Teramoto, A.; Nakamura, N.; Fujiki, M.; Sato, T. Macromolecules 2001, 34, 6519-6525.

(31) Peeters, E.; Delmotte, A.; Janssen, R. A.; Meijer, E. W. Adv. Mater. 1997, 9, 493-496.

(32) Fujiki, M. J. Am. Chem. Soc. 1996, 118, 7424-7425.

(33) Natsume, T.; Wu, L.; Sato, T.; Terao, K.; Teramoto, A.; Fujiki, M. Macromolecules 2001, 34, 7899-7904.

(34) Fujiki, M. J. Am. Chem. Soc. 1994, 116, 6017-6018.

(35) Indovina, P. L.; Tettamanti, E.; Micciancio-Giammarinaro, M. S.; Palma, M. U. J. Chem. Phys. 1979, 70, 2841-2847.

(36) Nishinari, K.; Koide, S.; Williams, P. A.; Phillips, G. O. J. Phys. (Paris) 1990, 51, 1759-1768.

(37) Izumi, Y.; Takezawa, H.; Kikuta, N.; Uemura, S.; Tsutsumi, A. Macromolecules 1998, 31, 430435.

(38) Wu, C.; Wang, X. Phys. Rev. Lett. 1998, 80, 4092-4094.

(39) Wang, X.; Qiu, X.; Wu, C. Macromolecules 1998, 31, 2972-2976. 


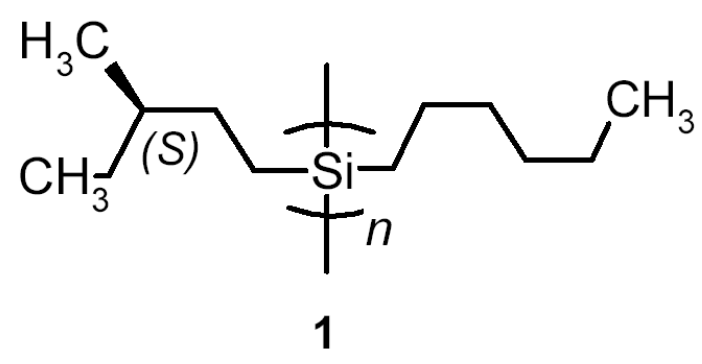

Scheme 1. Chemical structure of poly[n-hexyl-(S)-3-methylpentylsilane] (PH3MPS)

Table 1. Prepared microcapsules confining PH3MPS

\begin{tabular}{|c|c|c|c|c|c|c|}
\hline Series & \multicolumn{3}{|c|}{$\mathrm{MC} 15 \mathrm{k}$} & \multicolumn{3}{|c|}{ MC5k } \\
\hline Immersed rotation / rpm & \multicolumn{3}{|c|}{15000} & \multicolumn{3}{|c|}{5000} \\
\hline Average outer diameter/ $\mu \mathrm{m}$ & \multicolumn{3}{|c|}{1.4} & \multicolumn{3}{|c|}{2} \\
\hline$c_{\mathrm{p}} / 10^{-3} \mathrm{~g} \mathrm{~cm}^{-3}$ & Sample & $m_{\mathrm{p}} / 10^{-14} \mathrm{~g}$ & $n_{\mathrm{p}} / 10^{4}$ & Sample & $m_{\mathrm{p}} / 10^{-14} \mathrm{~g}$ & $n_{\mathrm{p}} / 10^{4}$ \\
\hline 0.20 & MC15k-02 & $0.020_{9}$ & $0.19_{1}$ & MC5k-02 & $0.061_{1}$ & $0.55_{7}$ \\
\hline 0.60 & MC15k-06 & $0.063_{0}$ & $0.57_{3}$ & MC5k-06 & $0.18_{3}$ & $1.6_{7}$ \\
\hline 2.0 & MC15k-2 & $0.20_{9}$ & $1.9_{1}$ & MC5k-2 & $0.61_{1}$ & $5.5_{7}$ \\
\hline 6.0 & MC15k-6 & $0.63_{0}$ & $5.7_{3}$ & MC5k-6 & 1.83 & 16.7 \\
\hline
\end{tabular}

\section{Figure captions}

Figure 1. Schematic representation to exchange the inner medium in microcapsules.

Figure 2. Differential UV absorption spectra for the indicated samples in tetrahydrofuran at $40^{\circ} \mathrm{C}$.

Figure 3. $\mathrm{CD}$ spectra for $\mathrm{MC} 15 \mathrm{k}$ prepared in ethanol at $T_{\mathrm{p}}=-78^{\circ} \mathrm{C}$ and measured at $T_{\mathrm{m}}=20^{\circ} \mathrm{C}$. Open circles, MC15k-02; filled circles, MC15k-06; open triangles, MC15k-2; filled triangles, MC15k-6.

Figure 4. Plots of $\triangle C D$ vs polymer concentration $c_{\mathrm{p}}$ in microcapsules and average polymer mass $m_{\mathrm{p}}$ per microcapsule for (a) $\mathrm{MC} 15 \mathrm{k}$ and (b) $\mathrm{MC} 5 \mathrm{k}$ in ethanol at $T_{\mathrm{m}}=20^{\circ} \mathrm{C}$. Open circles, $T_{\mathrm{p}}=-78^{\circ} \mathrm{C}$; filled circles, $T_{\mathrm{p}}=20^{\circ} \mathrm{C}$.

Figure 5. Circular dichroism for MC15k-6 at $T_{\mathrm{p}}=20^{\circ} \mathrm{C}$. (a) $\mathrm{CD}$ spectra at $T_{\mathrm{m}}=20^{\circ} \mathrm{C}$ (triangles) and $0^{\circ} \mathrm{C}$ (stepwise cooling from $20^{\circ} \mathrm{C}$, open circles; directly cooling from $20^{\circ} \mathrm{C}$, filled circles). (b) $\triangle C D$ on cooling process from $T_{\mathrm{m}}=20^{\circ} \mathrm{C}$ to $-10^{\circ} \mathrm{C}$; the symbols are the same as those in panel (a). (c) $\triangle C D$ in multiple thermal cycles of cooling to $0^{\circ} \mathrm{C}$ and heating to $20^{\circ} \mathrm{C}$.

Figure 6. Circular dichroism for MC15k-6 prepared at $T_{\mathrm{p}}=20^{\circ} \mathrm{C}$ on cooling process from $T_{\mathrm{m}}=40$ to $0^{\circ} \mathrm{C}$. (a) $\mathrm{CD}$ spectra at $40^{\circ} \mathrm{C}$ (triangles) and $0^{\circ} \mathrm{C}$ (stepwise cooling from $40^{\circ} \mathrm{C}$, open circles; directly cooling from $40^{\circ} \mathrm{C}$, filled circles). (b) Temperature dependence of $\triangle C D$; the symbols are the same as those in panel (a).

Figure 7. Plots of $\triangle C D$ vs average polymer concentration $c_{\mathrm{p}}$ in microcapsules and average polymer mass $m_{\mathrm{p}}$ per microcapsule for (a) MC15k and (b) $\mathrm{MC} 5 \mathrm{k}$ in tetrahydrofuran at $T_{\mathrm{m}}=20^{\circ} \mathrm{C}$ (open triangles, initial; filled triangles, first heating; open squares, second heating) and at $T_{\mathrm{m}}=0^{\circ} \mathrm{C}$ (open circles, first cooling; filled circles, second cooling). Temperature was changed as the following sequences: $20,-10$, $40,0,40^{\circ} \mathrm{C}$.

Figure 8. Temperature dependence of $\Delta \Delta \varepsilon$ for MC15k-6 (filled triangles), MC15k-2 (open triangles), MC15k-06 (filled circles), and MC15k-02 (open circles) prepared at $T_{\mathrm{p}}=20^{\circ} \mathrm{C}$ in tetrahydrofuran; (a) first cooling from $20^{\circ} \mathrm{C}$ and first heating, (b) second cooling from $40^{\circ} \mathrm{C}$ and heating. 


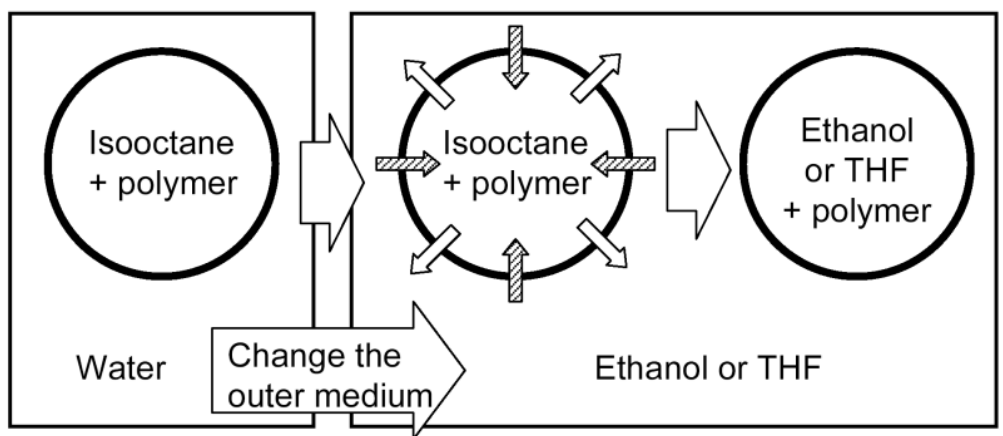

Figure 1

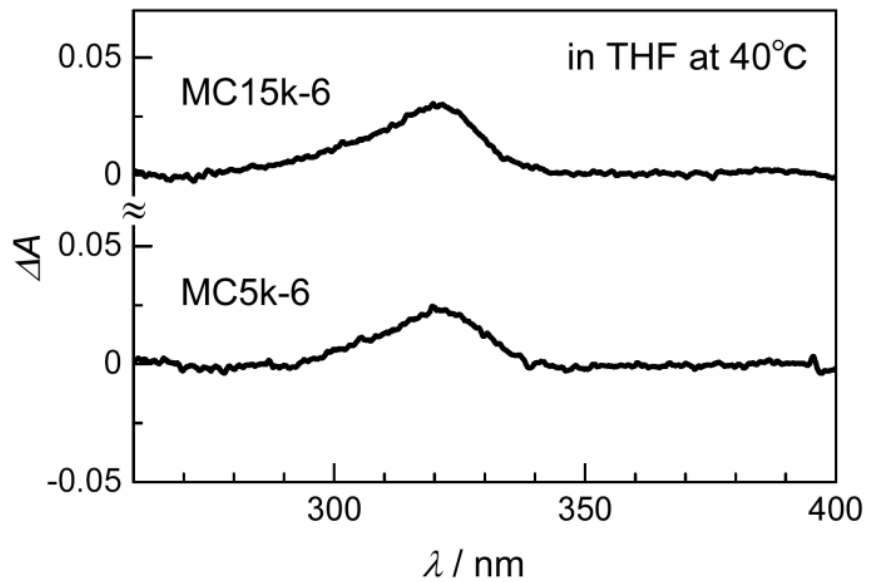

Figure 2 


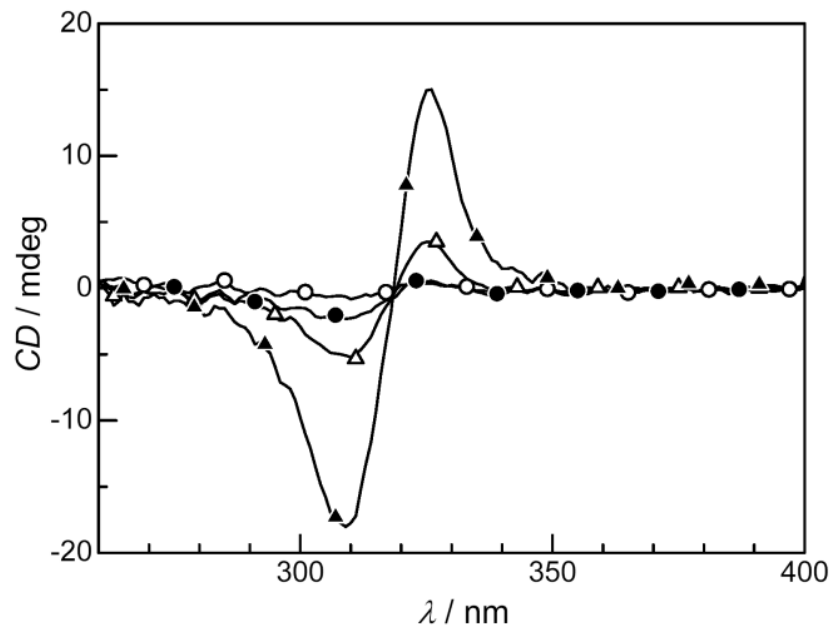

Figure 3

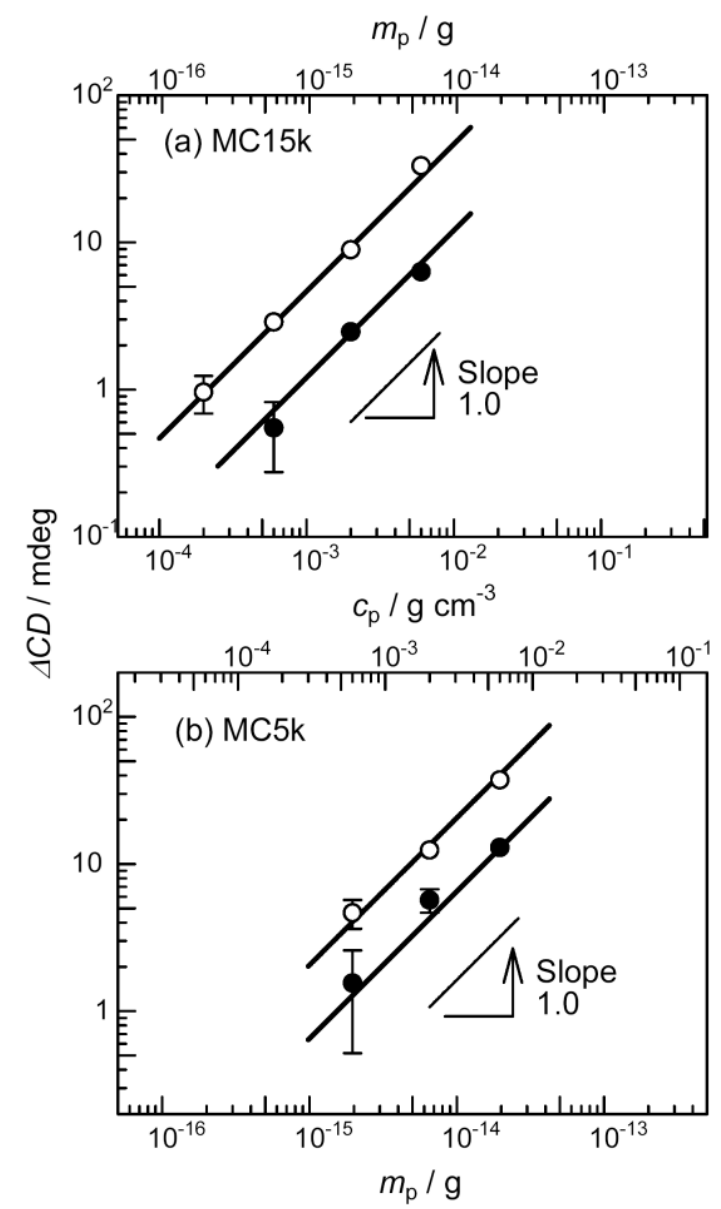

Figure 4 


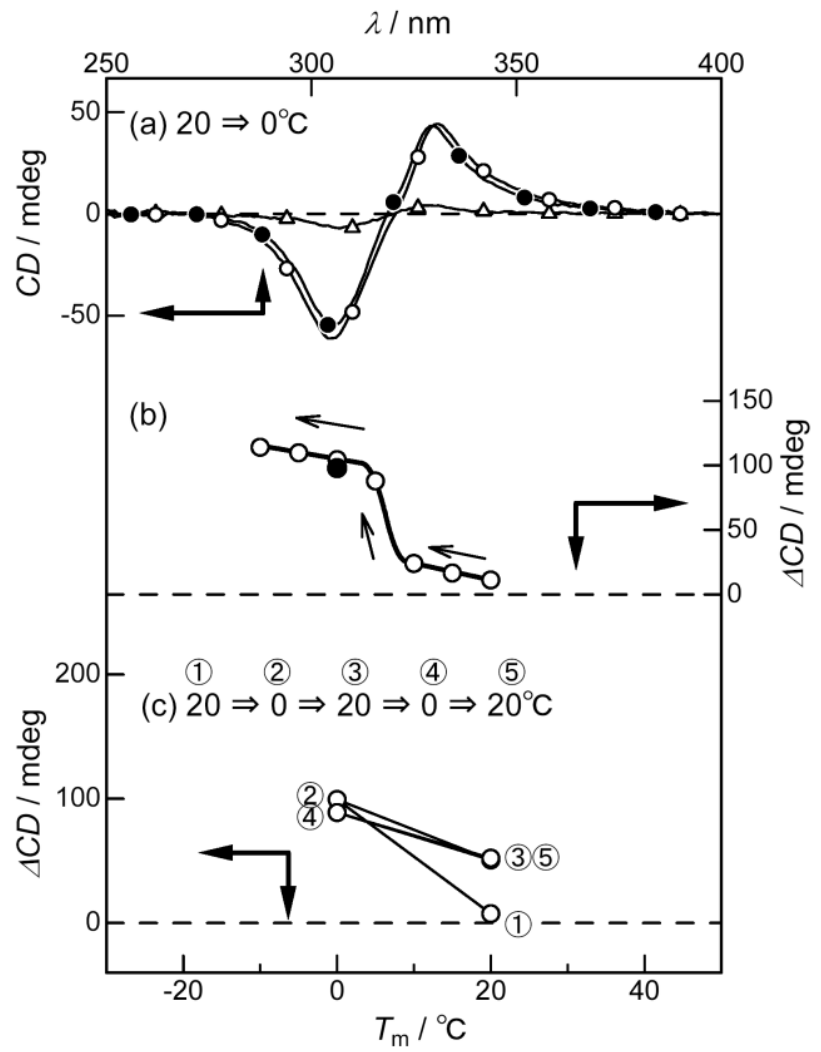

Figure 5

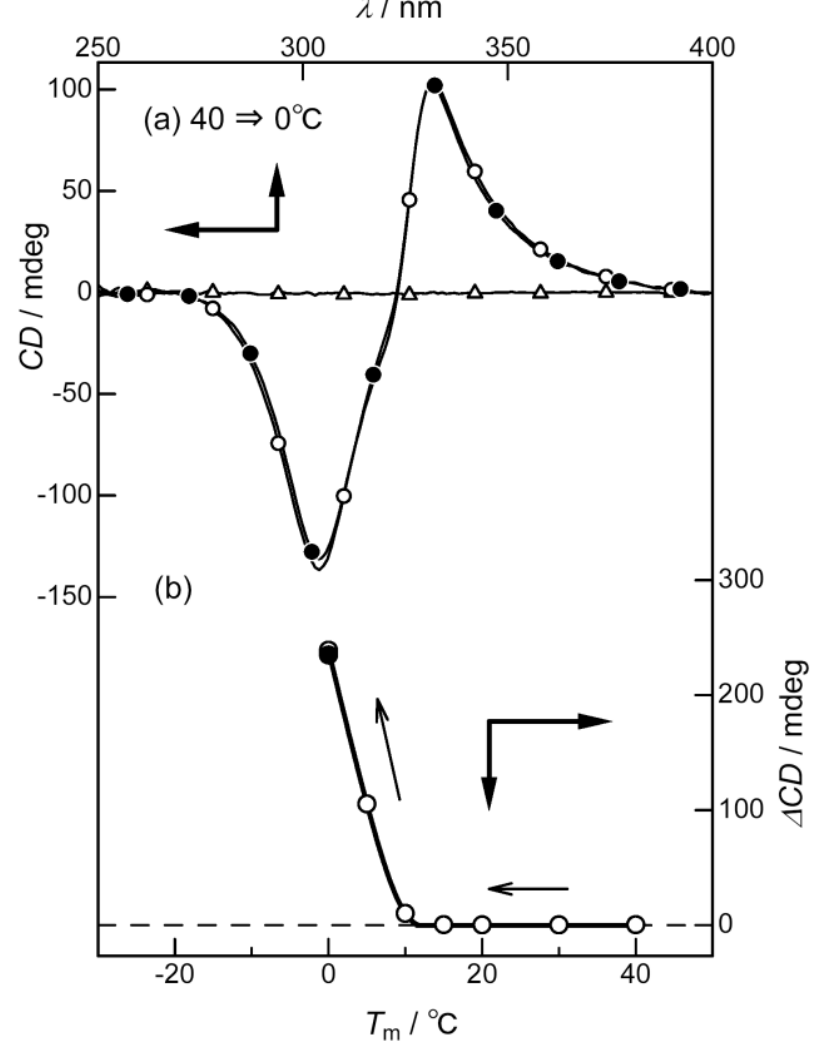

Figure 6 

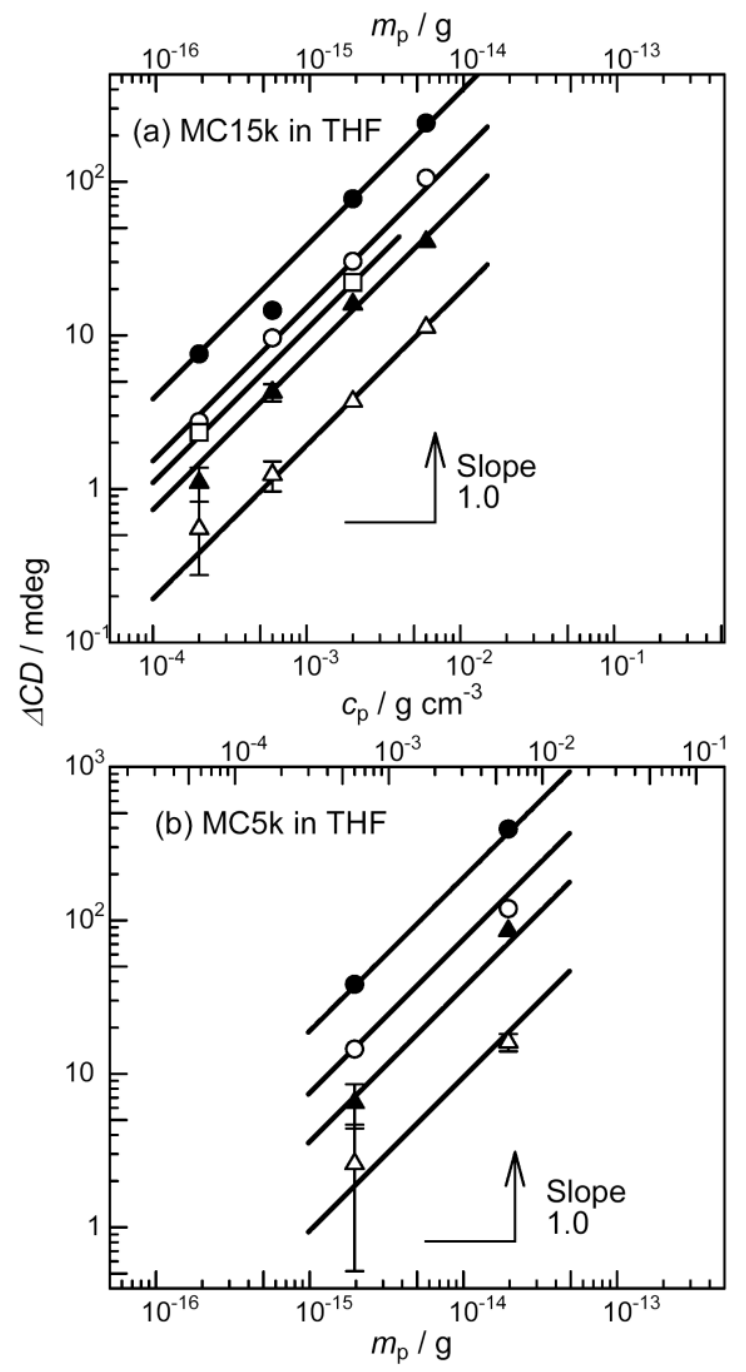

Figure 7

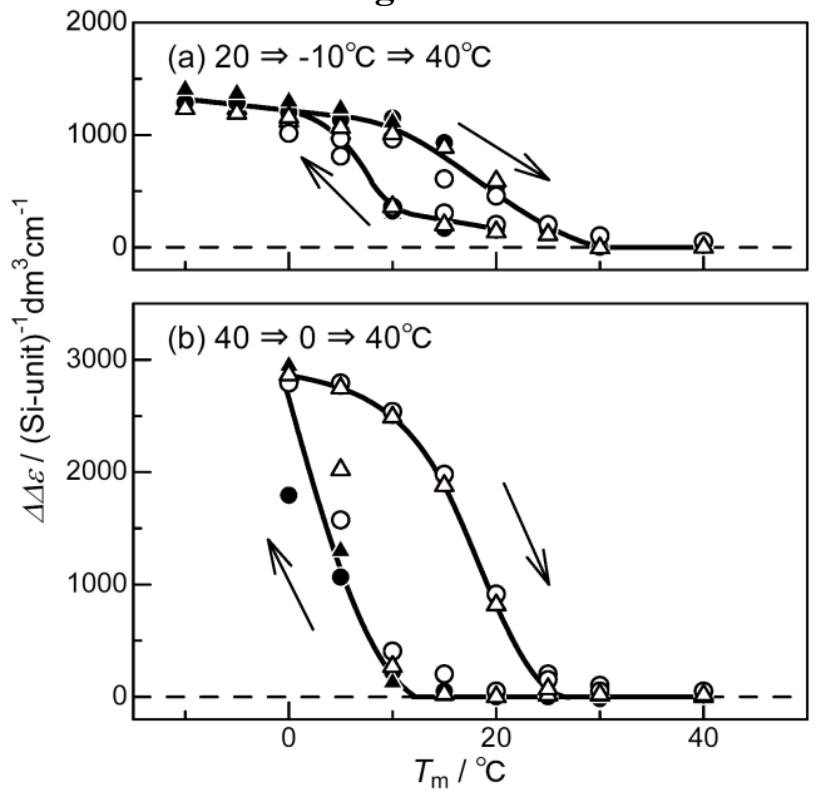

Figure 8 

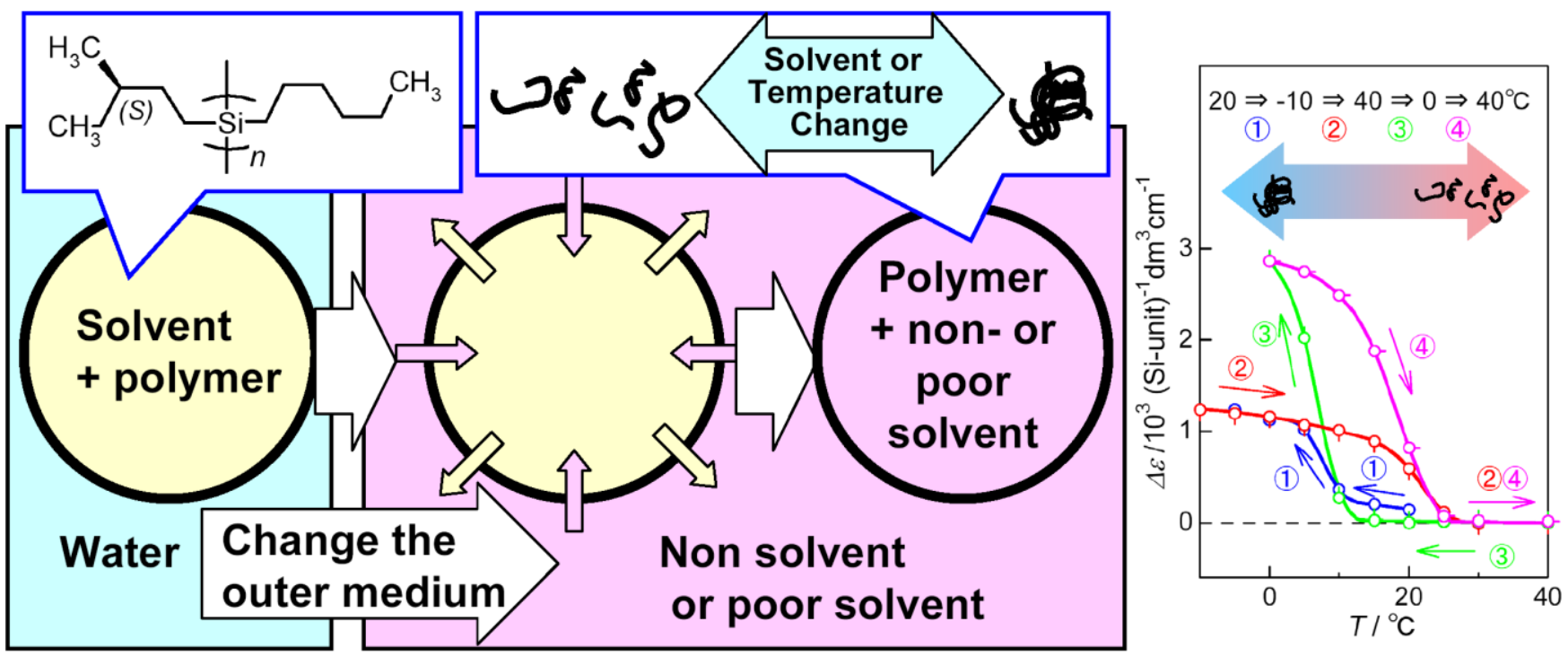

Table of Contents 